\title{
Precipitation and evolution of nodular graphite during solidification process of ductile iron
}

\author{
Jin-hai Liu, ${ }^{1,2}$, Jian-shuai Yan', Xue-bo Zhao', *Bin-guo Fu ${ }^{1,2}$, Hai-tao Xue', Gui-xian Zhang, ${ }^{2,3}$ and Peng-hui Yang ${ }^{4}$ \\ 1. School of Materials Science and Engineering, Hebei University of Technology, Tianjin 300130, China \\ 2. Key Laboratory of Research and Application of Mould Materials for Glass and Rubber in Hebei Province, Huanghua 061000, China \\ 3. Hebei Andi Mould Co., Ltd, Huanghua 061000, China \\ 4. School of Materials Science and Engineering, Beijing University of Technology, Beijing 100124, China
}

\begin{abstract}
The quantity and morphology of spheroidal graphite have an important effect on the properties of ductile iron, and the characteristics of spheroidal graphite are determined by the solidification process. The aim of this work is to explore the precipitation and evolution of graphite nodules in hypoeutectic, eutectic, and hypereutectic ductile irons by thermal analysis, liquid quenching and metallographic technique. Results show that hypoeutectic ductile iron has the longest solidification time and the lowest eutectic temperature; eutectic ductile iron has the shortest solidification time; hypereutectic ductile iron has the highest eutectic temperature. After solidification is completed, hypoeutectic ductile iron has the lowest nodule count, nodularity and graphite fraction; eutectic ductile iron has the highest nodule count, nodularity and the smallest nodule diameter; hypereutectic has the highest nodule diameter and graphite fraction. The nucleation and growth of graphite nodules in hypereutectic ductile iron starts before bulk eutectic crystallization stage, however, the precipitation and evolution of graphite nodules of hypoeutectic and eutectic ductile irons mainly occur in the eutectic crystallization stage. The graphite precipitated in eutectic crystallization of hypoeutectic, eutectic, and hypereutectic ductile irons, are $61 \%, 68 \%$ and $43 \%$ of total graphite volume fraction, respectively. Simultaneously, there are plenty of austenite dendrites in hypoeutectic and hypereutectic ductile irons, which are prone to shrinkage defects. Therefore, the eutectic ductile iron has the smallest shrinkage tendency.
\end{abstract}

Key words: ductile iron; carbon equivalent; graphite nodules; solidification; thermal analysis; liquid quenching
CLC numbers: TG143.5
Document code: A
Article ID: 1672-6421 202004-260-12

$\mathrm{D}$ uctile iron is an indispensable engineering material with excellent mechanical performances. Its tensile strength is even comparable to that of steel because the existence of graphite in a spherical shape reduces the cleavage effect of stress concentration in the matrix ${ }^{[1,2]}$. In recent years, it has been found that austempered ductile iron (ADI) and carbidic austempered ductile iron (CADI), which are obtained by the austempering process, have higher strength and shock absorption, as well as good wear resistance and plastic toughness, giving rise to ductile iron being more widely used ${ }^{[3-5]}$. The quantity and morphology of graphite have a great influence on the properties of ductile iron. For examples, graphite nodules with small diameter and high roundness can significantly improve

\section{*Bin-guo Fu}

Male, born in 1984, Ph.D. His research interests mainly focus on the cast iron melt quality online measurement and control.

E-mail: fubinguohao@163.com

Received: 2020-03-24; Accepted: 2020-05-11 the mechanical properties of castings ${ }^{[6,7]}$, and with the increase of nodules, the risk of carbide precipitation decreases and the matrix performance improves significantly ${ }^{[8]}$. The increase of nodules will also make the eutectic grains profile more round which is conducive to the flow filling of melt, so that the shrinkage tendency of ductile iron will be reduced ${ }^{[9]}$. The characteristics of graphite are determined by the solidification process of ductile iron ${ }^{[10]}$. However, the solidification process of ductile iron is complex, and the transformation mechanism of the structure in the mushy zone have not been yet mastered ${ }^{[11,12]}$. Therefore, it is necessary to do a further study on the precipitation and evolution of spheroidal graphite during the solidification of ductile iron.

Liquid quenching is an effective method to study the solidification behaviors and characteristics of cast iron. By quenching the melt, the microstructure at high temperature can be quickly cooled and obtained, so the phase transformation during the solidification process could be studied ${ }^{[13,14]}$. In the 1960s, Oldfield first quantitatively studied the nucleation and growth 
of eutectic grains during the solidification of gray cast iron by quenching semi-solid samples ${ }^{[15]}$. Since then, liquid quenching has been widely used to study the microstructure evolution of the cast iron during the solidification process. Additionally, thermal analysis is a significant tool in the casting production process. During the solidification of cast iron, latent heat will be released when the liquid phase transforms to the solid phase, which will change the slope of the thermal analysis curve of the melt. Therefore, the structural changes can be understood ${ }^{[16]}$. Combining thermal analysis with liquid quenching can obtain a solidified microstructure at a specific time of solidification, and it can be used to study the solidification process of cast iron more accurately and efficiently. By this way, Stefanescu ${ }^{[17,18]}$ quenched different Mg-containing melts, and systematically studied the growth mechanism and shape change of vermicular graphite; Natxiondo A et al. ${ }^{[13,19]}$ investigated the effects of inoculation and carbon content on graphite nucleation and growth during the solidification of hypoeutectic ductile iron; Fredriksson ${ }^{[20]}$ studied the microstructure evolution of the hypoeutectic ductile iron during solidification and explained the formation mechanism of shrinkage porosity.

Carbon content, alloying elements, and inoculation can affect the solidification characteristics of ductile iron, with the carbon content playing a decisive role. According to the different carbon content, ductile iron is divided into hypoeutectic, eutectic, and hypereutectic, which have obvious differences in their solidification processes ${ }^{[1]}$. In practical production, the composition of ductile iron is usually selected as eutectic or hypereutectic. However, past research on the solidification of ductile iron mostly focuses on the hypoeutectic composition. In this study, three kinds of ductile irons with different carbon contents were prepared, and the precipitation and evolution of graphite nodules in hypoeutectic, eutectic and hypereutectic ductile irons were systematically investigated by thermal analysis, liquid quenching and metallographic technique. In addition, the effect of eutectic graphite precipitation on shrinkage tendency of the ductile iron was also discussed.

\section{Experimental procedure}

\subsection{Liquid quenching experiment}

The experimental ductile irons were prepared by a $35 \mathrm{~kg}$ medium frequency induction furnace. The raw materials were pig iron, scrap steel, ferrosilicon and ferromanganese. A NSP3601 carbon-silicon analyzer was used to adjust the carbon and silicon composition of the melt. After the carbon and silicon content of the melt was adjusted to the target range, the melt was heated to $1,550{ }^{\circ} \mathrm{C}$ and held for $2 \mathrm{~min}$, and then spheroidized. The spheroidizing treatments were carried out by the sandwich method: the melt was poured into the ladle, which contains $455 \mathrm{~g}$ of FeSiMg6RE2 nodulizer, $175 \mathrm{~g}$ of inoculant and $350 \mathrm{~g}$ of steel sheet.

A DX104 data collection instrument was used to obtain thermal analysis curves. The instrument has the functions of amplification, filtering, and A/D conversion, and can record the received signals and transmit them to a computer. The sampling cups used in the experiment are made of resin sand, which have a lid with two holes that can control the flow rate of the melt, ensuring a uniform sample volume. A K-type thermocouple is set in the center to collect the temperature signals. Figure 1 shows the internal appearance of the sampling cup.

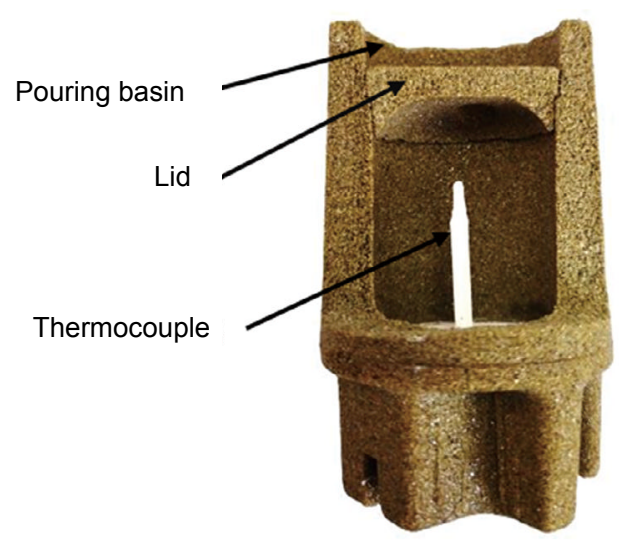

Fig. 1: Sampling cup

After spheroidizing treatment, slag scraping was performed, and then the melt was quickly poured into quenching sample cups and shrinkage sample cups, which are same as the liquid quenching sample cups except that they have no riser and thermocouple. The pouring temperature was controlled between 1,400 to $1,410{ }^{\circ} \mathrm{C}$. When the temperature on the thermal analysis curve reached the expected liquid quenching point (Fig. 2), a sample was quickly placed into salt water of $0-5{ }^{\circ} \mathrm{C}$, while the water is rapidly stirred to accelerate the sample cooling. A sample was retained in the sample cup holder to record the whole cooling curve. The definition of the characteristic values of thermal analysis curve are shown in Table 1. The chemical composition of the ductile iron in this experiment is shown in Table 2. It can be seen that the carbon equivalent (CE) of eutectic ductile iron is $4.50 \mathrm{wt} . \%$, which is far above the expected $\mathrm{CE}$ at $4.26 \mathrm{wt} . \%-4.34 \mathrm{wt} . \%$. This is because the spheroidizing elements $(\mathrm{Mg}, \mathrm{Ce})$ in ductile iron will increase the degree of undercooling during the solidification process, which will increase the eutectic carbon equivalent of ductile iron.

\subsection{Metallographic analysis}

In the quenching process, the phases in the solidifying sample changed as follows: there was no change of the graphite that had been precipitated in liquid, the austenite transformed into martensite or pearlite, and the unsolidified liquid was chilled into ledeburite, simultaneously. Very small chilled graphite spheres were also precipitated.

After cooling, the pouring basins of the samples were removed, and then the sample was cut longitudinally with an electric spark cutting machine. The microstructure of the sample was analyzed by an optical microscope after being etched with $4 \%$ nital solution. The area of analysis is as shown in Fig. 3. Image analysis software was used to measure 
(a)

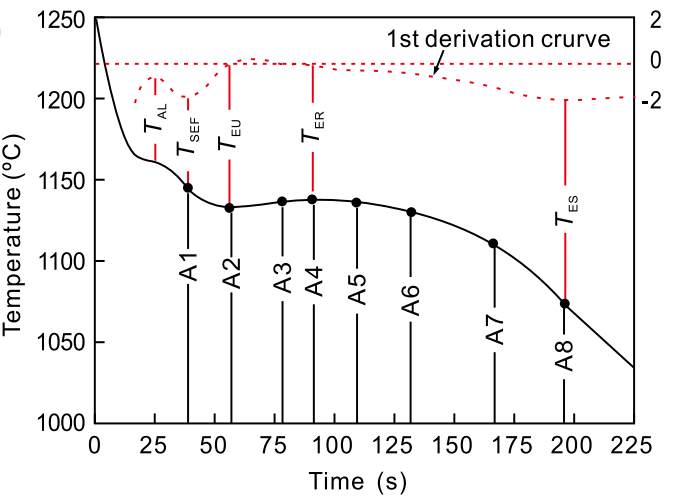

(b)

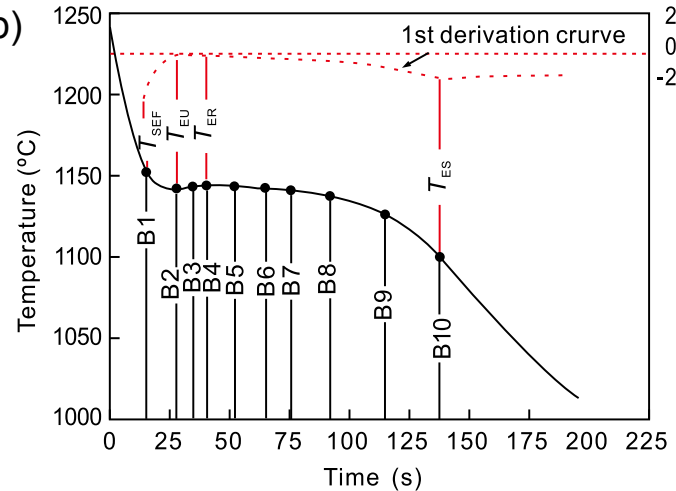

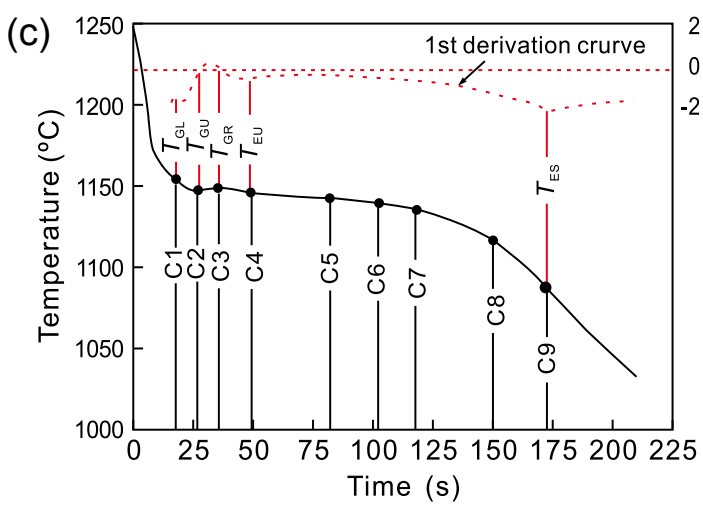

Fig. 2: Liquid quenching sequence of three kinds of ductile iron: (a) A (hypoeutectic); (b) B (eutectic); (c) C (hypereutectic)

Table 1: Definition of characteristic values of thermal analysis curves ${ }^{[21]}$

\begin{tabular}{|c|c|}
\hline Characteristic value & Significance \\
\hline$T_{\mathrm{AL}}$ & Temperature of commencement of primary austenite precipitation \\
\hline$T_{\mathrm{SEF}}$ & Temperature of the start of eutectic freezing \\
\hline$T_{\mathrm{GL}}$ & Temperature fluctuation caused by primary graphite precipitation \\
\hline$T_{\mathrm{GU}}$ & $\begin{array}{l}\text { Minimum temperature of pro-eutectic austenite precipitation in } \\
\text { hypereutectic ductile iron }\end{array}$ \\
\hline$T_{\mathrm{GR}}$ & $\begin{array}{l}\text { Maximum temperature of pro-eutectic austenite precipitation in } \\
\text { hypereutectic ductile iron }\end{array}$ \\
\hline$T_{\mathrm{EU}}$ & Temperature of bulk eutectic initiation \\
\hline$T_{\mathrm{ER}}$ & The highest eutectic temperature \\
\hline$T_{\mathrm{ES}}$ & Temperature of the end of solidification \\
\hline
\end{tabular}

Table 2: Chemical composition of ductile irons in this experiment (wt.\%)

$\begin{array}{ccccccccc}\text { Alloy } & \text { C } & \text { Si } & \text { CE } & \text { Mn } & \text { P } & \text { S } & \text { Mg } \\ \text { A (hypoeutectic) } & 3.45 & 2.57 & 4.31 & 0.324 & 0.028 & 0.0172 & 0.047 \\ \text { B (eutectic) } & 3.65 & 2.55 & 4.50 & 0.318 & 0.026 & 0.0145 & 0.051 \\ \text { C (hypereutectic) } & 3.80 & 2.65 & 4.68 & 0.314 & 0.028 & 0.0128 & 0.048\end{array}$

Carbon equivalent calculated as $\mathrm{CE}=\mathrm{C}+\mathrm{Si} / 3$ 
the nodule count, average diameter, nodularity (percentage of number of spheroidal graphite in total graphite) and the graphite fraction of samples according to the standard of GB/T 9441-2009 (Equivalent to ISO 945-1) ${ }^{[22]}$. Five micrographs in the center of the samples were selected for calculation, and the average value was taken as the final result. Liquid-quenching will form chilled graphite which would not be surrounded by austenitic shells, but would always be in contact with the liquid phase. In general, the diameter of this type graphite would not exceed $15 \mu \mathrm{m}{ }^{[23]}$. Therefore, the graphite spheres with a diameter less than $20 \mu \mathrm{m}$ were ignored to avoid the effect of chilled graphite spheres on statistical results.
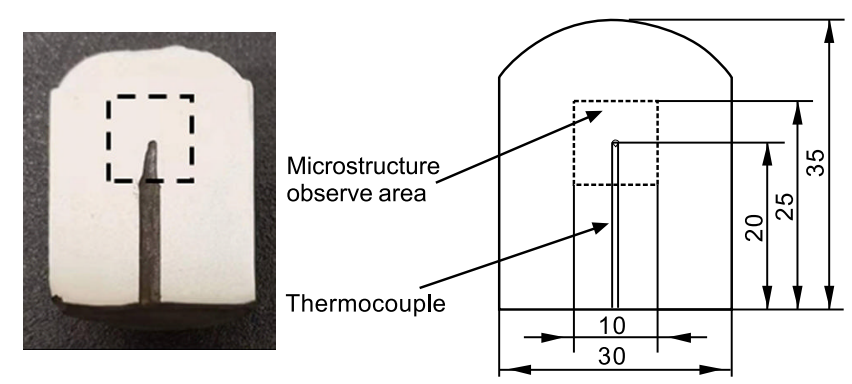

Fig. 3: Microstructure analysis area of quenched sample (unit: $\mathrm{mm}$ )

\section{Results and analysis}

\subsection{Thermal analysis curves}

Figure 4 indicates the thermal analysis curves of the three kinds of ductile irons. It can be seen that the precipitation of primary austenite leads to an inflection point $\left(T_{\mathrm{AL}}\right)$ in the hypoeutectic thermal analysis curve. The thermal analysis curve of eutectic ductile iron has no inflection point before the eutectic recalescence. For hypereutectic ductile iron, the primary graphite precipitation caused the $T_{\mathrm{GL}}$ point, and then the austenite begun to form due to the non-equilibrium

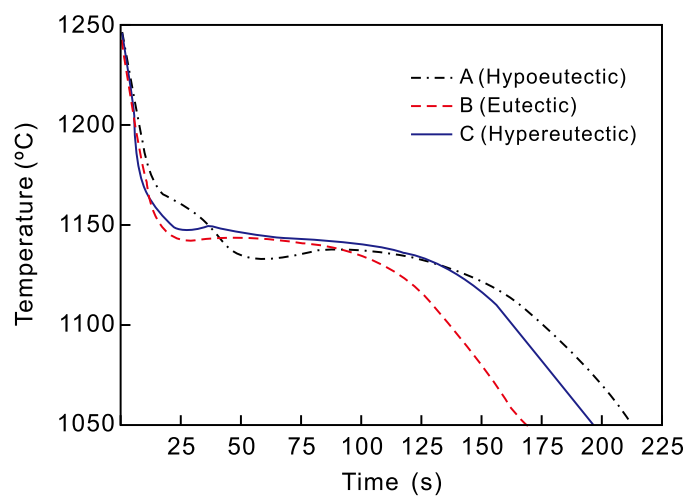

Fig. 4: Thermal analysis curves obtained from experiment

crystallization. After that, the temperature reaches the highest point $\left(T_{\mathrm{GR}}\right)$ because the releasing of latent heat. Subsequently, the eutectic solidification occurred.

Table 3 shows the characteristic values of the curves in Fig. 4. It can be found that the eutectic temperature of hypereutectic ductile iron is the highest, and the hypoeutectic ductile iron is the lowest. During the solidification process of hypereutectic ductile iron, primary graphite nodules first precipitated at high temperature. These nodules can act as the nucleus substrates of eutectic reaction ${ }^{[1]}$. Therefore, the eutectic crystallization of hypereutectic ductile iron does not need a large undercooling, as the temperature of eutectic stage is the highest. In order to ensure the progress of the eutectic reaction, further undercooling is needed, so there is no recalescence on the thermal analysis curve. Unlike a hypereutectic melt, the carbon content of hypoeutectic ductile iron is low and a certain amount of austenite dendrites are formed before eutectic. So, only when the melt was undercooled largely enough, the kinetic conditions for the formation of eutectic graphite cores can be reached. The temperature of the hypoeutectic ductile iron eutectic stage is relatively low.

The solid phase (graphite or austenite) of hypereutectic, hypoeutectic and eutectic ductile irons was first precipitated at $16 \mathrm{~s}, 26 \mathrm{~s}$ and $28 \mathrm{~s}$, respectively. The total freezing time of

Table 3: Characteristic values of thermal analysis curves

\begin{tabular}{|c|c|c|c|c|c|c|c|c|c|c|c|}
\hline \multicolumn{2}{|c|}{ Alloy } & $T_{\mathrm{GL}}$ & $T_{\mathrm{AL}}$ & $T_{\mathrm{GU}}$ & $T_{\mathrm{GR}}$ & $T_{\mathrm{SEF}}$ & $T_{\mathrm{EU}}$ & $T_{\text {ER }}$ & $T_{\mathrm{ES}}$ & $\begin{array}{l}\text { Solidifying } \\
\text { time }\end{array}$ & $\begin{array}{l}\text { Percentage of } \\
\text { eutectic period }\end{array}$ \\
\hline \multirow{2}{*}{$\begin{array}{c}\text { A } \\
\text { (Hypoeutectic) }\end{array}$} & $\begin{array}{l}\text { Temperature } \\
\left({ }^{\circ} \mathrm{C}\right)\end{array}$ & - & 1,161 & - & - & 1,147 & 1,133 & 1,137 & 1,076 & - & - \\
\hline & Time (s) & - & 26 & - & - & 37 & 56 & 93 & 196 & 170 & $82 \%$ \\
\hline \multirow{2}{*}{$\begin{array}{c}\mathrm{B} \\
\text { (Eutectic) }\end{array}$} & $\begin{array}{l}\text { Temperature } \\
\left({ }^{\circ} \mathrm{C}\right)\end{array}$ & - & - & - & - & 1,156 & 1,142 & 1,144 & 1,090 & - & - \\
\hline & Time (s) & - & - & - & - & 15 & 28 & 42 & 136 & 121 & $100 \%$ \\
\hline \multirow{2}{*}{$\frac{\text { C }}{\text { (Hypereutectic) }}$} & $\begin{array}{c}\text { Temperature } \\
\left({ }^{\circ} \mathrm{C}\right)\end{array}$ & 1,155 & - & 1,147 & 1,149 & - & 1,147 & - & 1,086 & - & - \\
\hline & Time (s) & 16 & - & 27 & 34 & - & 53 & - & 173 & 157 & $76 \%$ \\
\hline
\end{tabular}


hypereutectic, hypoeutectic and eutectic iron is $157 \mathrm{~s}, 170 \mathrm{~s}$, and $121 \mathrm{~s}$, respectively. Meanwhile, the proportion of eutectic time in total freezing time is $76 \%, 82 \%$ and $100 \%$, respectively, for hypereutectic, hypoeutectic and eutectic ductile irons. Because the eutectic ductile iron has no primary phase precipitation, the eutectic crystallization starts early and goes fast, so that the time to reach the $T_{\mathrm{ES}}$ point is shorter and the $T_{\mathrm{ES}}$ temperature is higher. For hypoeutectic and hypereutectic ductile irons, the primary phase nucleates and grows in hightemperature liquid before eutectic crystallization stage, and the great latent heat can't be quickly transferred to the external environment, resulting in a slower solidification speed, and the eutectic crystallization is delayed. Simultaneously, a large amount of primary phase precipitation leads to a decrease of liquid volume used for eutectic, so the eutectic latent heat is low, and the sample temperature decreases rapidly in the last period of solidification. Therefore, the end temperature of eutectic solidification $\left(T_{\mathrm{ES}}\right)$ of hypoeutectic and hypereutectic ductile irons is lower than eutectic ductile iron.

\subsection{Evolution of microstructure during solidification}

\subsubsection{Solidification of hypoeutectic ductile iron}

Figure 5 shows the microstructures of liquid-quenched samples of hypoeutectic ductile iron at different solidifying periods. The microstructure of sample quenched at above $T_{\mathrm{SEF}}$ (A1) consists of fine austenite dendrites and small graphite spheres. Of course, there may be some chilled graphite nodules caused by liquid quenching, but a few of the nodular graphite were surrounded by thin austenite shells (Fig. 5a). The graphite spheres formed and grew in the hypoeutectic melt above the $T_{\mathrm{EU}}$ temperature and were caused by the constituent and temperature fluctuations ${ }^{[24]}$.
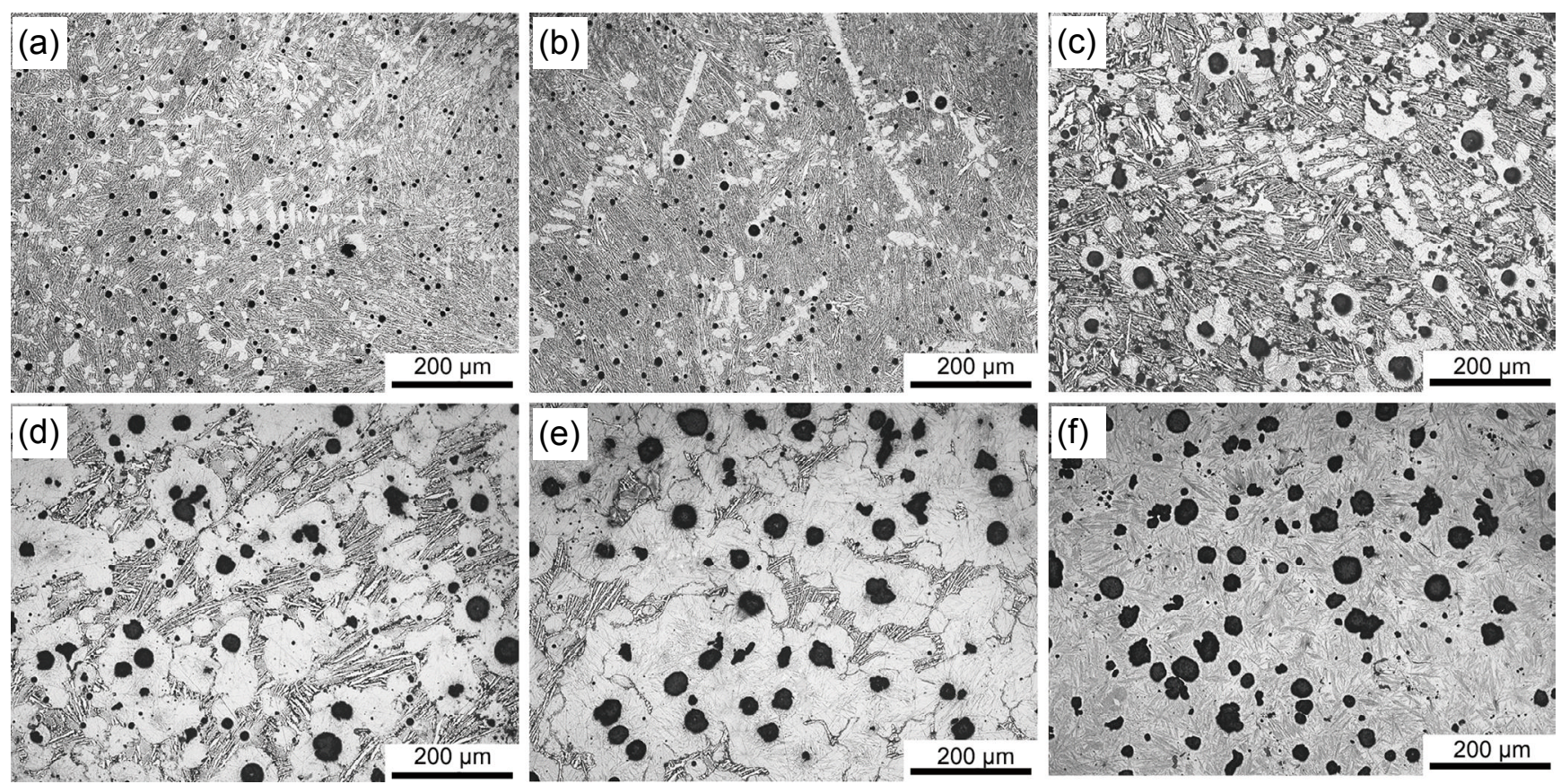

Fig. 5: Microstructure observed in liquid-quenched samples of $A$ (Hypoeutectic): (a) A1, $T_{\mathrm{SEF}}$; (b) A2, $T_{\mathrm{EU}}$; (c) A4, $37 \mathrm{~s}$ after $T_{\mathrm{EU}}\left(T_{\mathrm{ER}}\right)$; (d) A5, $53 \mathrm{~s}$ after $T_{\mathrm{EU}}$; (e) A7, $109 \mathrm{~s}$ after $T_{\mathrm{EU}}$; (f) A8, $140 \mathrm{~s}$ after $T_{\mathrm{EU}}\left(T_{\mathrm{ES}}\right)$

As the temperature decreased to $T_{\mathrm{EU}}$, the primary and secondary dendrites of austenite grew significantly, and some graphite spheres grew up, and the austenite shell around them became thicker (Fig. 5b). Meanwhile, because of the composition undercooling in the front of austenite, which caused the instability of the austenite growth, austenite dendrites would form and grow on the austenite shells ${ }^{[25]}$. It seems that some very small graphite spheres formed at high temperature liquid had disappeared because of the fading of inoculation, so only some of larger-sized graphite cores survived and grew up. Then, bulk eutectic crystallization began. Because the undercooling was large at $T_{\mathrm{EU}}$, the graphite nodules and austenite grew fast (Fig. 5c), the quantity of released latent heat was greater than heat transfer capacity, resulting in the recalescence (Fig. 4). As the solidification progressed, the diameter of the graphite nodules increased, the austenite shells around them became thicker and thicker. Since the diffusion rate of carbon atoms in the austenite is $1 / 20$ of that in the melt ${ }^{[26]}$, with the increase of the thickness of the austenite shells, the eutectic crystallization rate reduced, the release of latent heat became less. Therefore, the melt temperature gradually decreased.

From Fig. 5(d) and (e), it can be seen that the volume of liquid phase gradually decreased, the nodules and austenite dendrites grew further, and they contacted and merged with each other to form large-sized grains. Eutectic grains also met and merged with each other. At the same time, some of the austenite dendrites and shells gradually disappeared. Additionally, it can be found that new graphite cores were also formed in the remaining liquid phase after $T_{\mathrm{ER}}$. This is because as the austenite shells around graphite nodules become thicker and thicker, it is difficult for carbon atoms in the unsolidified 
melt to diffuse into the surface of the graphite through the austenite shell. Simultaneously, the melt temperature becomes lower and the degree of undercooling increases, which results in the kinetics driving force for nucleation of fresh graphite. As a result, a small quantity of nodules formed in the remaining melt and grew until all liquid is solidified at $T_{\mathrm{ES}}$, as shown in Fig. 5(f); so, the number of small nodules was significantly increased compared with Fig. 5(e).

However, in the final stages of the solidification, if the inoculation is severely faded or the positive segregation of the anti-graphitizing element happens, the number of fresh graphite cores will reduce remarkably. The last remained melt will crystallize according to the metastable system ${ }^{[27]}$, and some cementite may form.

\subsubsection{Solidification of eutectic ductile iron}

Figure 6 shows the microstructures of liquid-quenched samples of eutectic ductile iron. When the eutectic melt dropped to $T_{\mathrm{EU}}$ temperature, a large number of graphite spheres formed [Fig. 6(a)]. However, the large sized nodules perhaps nucleated in the melt above the $T_{\mathrm{EU}}$ temperature. After $T_{\mathrm{EU}}$, the austenite nucleated on the surface of the large graphite spheres, and grew into circular shell, indicating that eutectic solidification had begun [Fig. 6(b)]. Under the action of supercooling, nodular graphite and austenite shell grew rapidly, and a small amount of austenite dendrites will be precipitated ${ }^{[27]}$. These result in great latent heat release and the temperature of the melt increasing to $T_{\mathrm{ER}}$. When the solidification process in $T_{\mathrm{ER}}$ point, the austenite shells thickened continually, and the growth rate of nodular graphite formed in the early stage was decreased. In order to maintain the eutectic crystallization, it is necessary to generate fresh nuclei of graphite in the unsolidified melt, as shown in Fig. 6(c), (d) and (e). At this stage, the volume of the retained liquid phase was decreasing, and the newly formed eutectic microstructure gradually reduced. Therefore, the released latent heat became less, and the melt temperature continued to decrease. At the last freezing region, the temperature was low and the undercooling was great, so that many fresh graphite spheres were formed. These small graphite spheres grew fast until the end of eutectic, as shown in Fig. 6(f). Compared with hypoeutectic ductile iron, eutectic ductile iron precipitated more graphite spheres in the late solidification period.
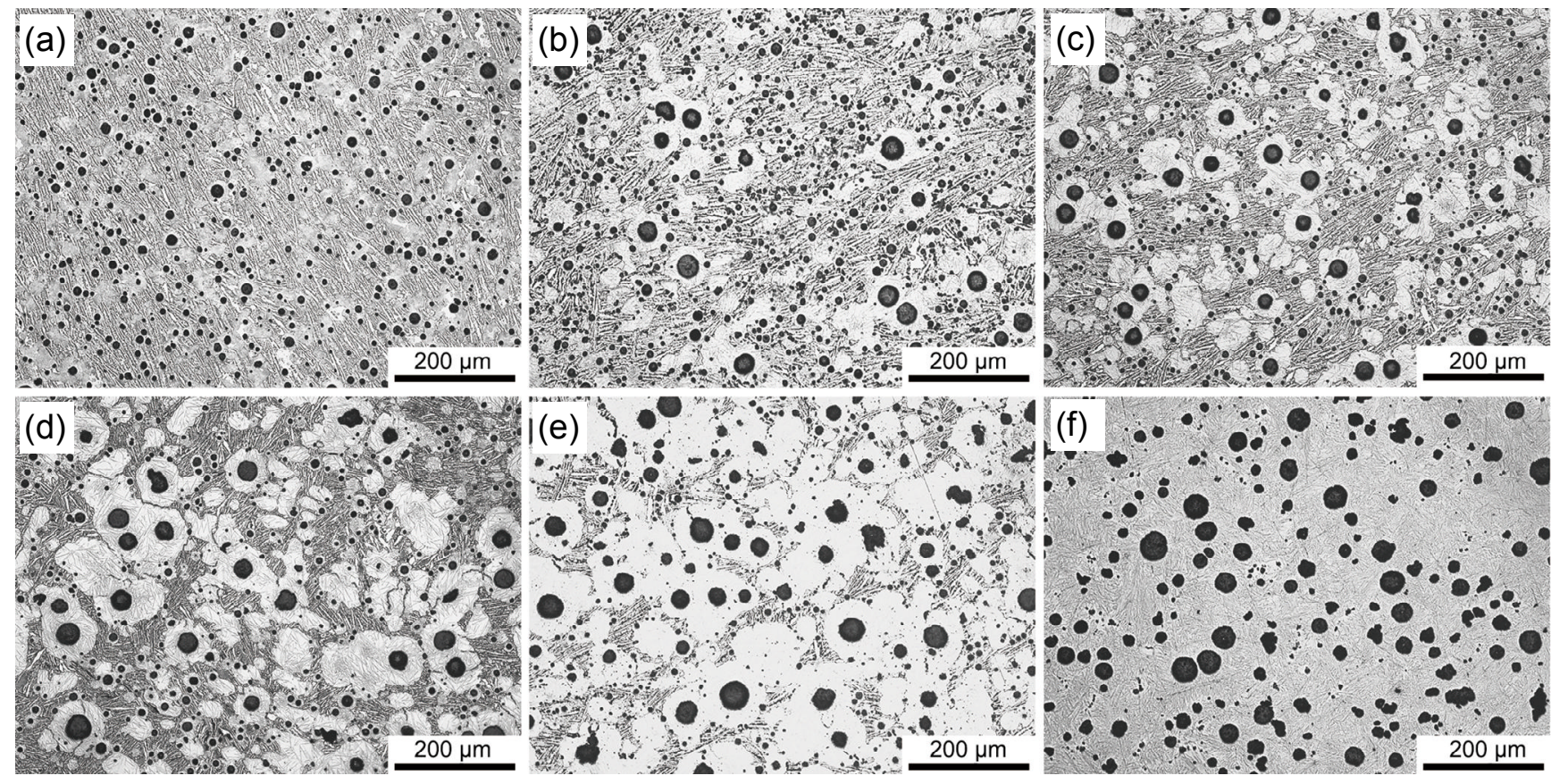

Fig. 6: Microstructures observed in liquid-quenched samples of $B$ (Eutectic): (a) B2, $T_{E U}$; (b) B4, $14 \mathrm{~s}$ after $T_{\mathrm{EU}}\left(T_{\mathrm{ER}}\right)$; (c) B5, $24 \mathrm{~s}$ after $T_{\mathrm{EU}}$; (d) B7, $47 \mathrm{~s}$ after $T_{\mathrm{EU}}$; (e) B9, $87 \mathrm{~s}$ after $T_{\mathrm{EU}}$; (f) B10, $108 \mathrm{~s}$ after $T_{\mathrm{EU}}\left(T_{\mathrm{ES}}\right)$

\subsubsection{Solidification of hypereutectic ductile iron}

Figure 7 shows the microstructures of liquid-quenched samples of hypereutectic ductile iron. It can be seen that the primary graphite precipitated and grew to a larger size in the melt above the $T_{\mathrm{GU}}$ temperature [Fig. 7(a)]. The rapid growth of primary graphite nodules in liquid caused a poor carbon liquid around them, which promoted the precipitation of the austenite shells and dendrites [Fig. 7(b)] ${ }^{[28,29]}$. Due to the rapid growth of austenite, a large amount of latent heat was released, and caused the emergence of extreme point $T_{\mathrm{GR}}$ on the thermal analysis curve. However, due to the surrounding by austenite shells, the growth rate of primary graphite reduced. At the same time, the growth of austenite dendrites caused rich carbon around its melt, which reduced the solidification rate of austenite. So, the melt temperature began to decrease again due to the decrease of latent heat. When the melt temperature dropped to about $T_{\mathrm{EU}}$, the bulk eutectic crystallization begun. Some new nodular graphite formed, as shown in Fig. 7(c), (d) and (e). During the late eutectic period, the austenite dendrites merged with the graphite 

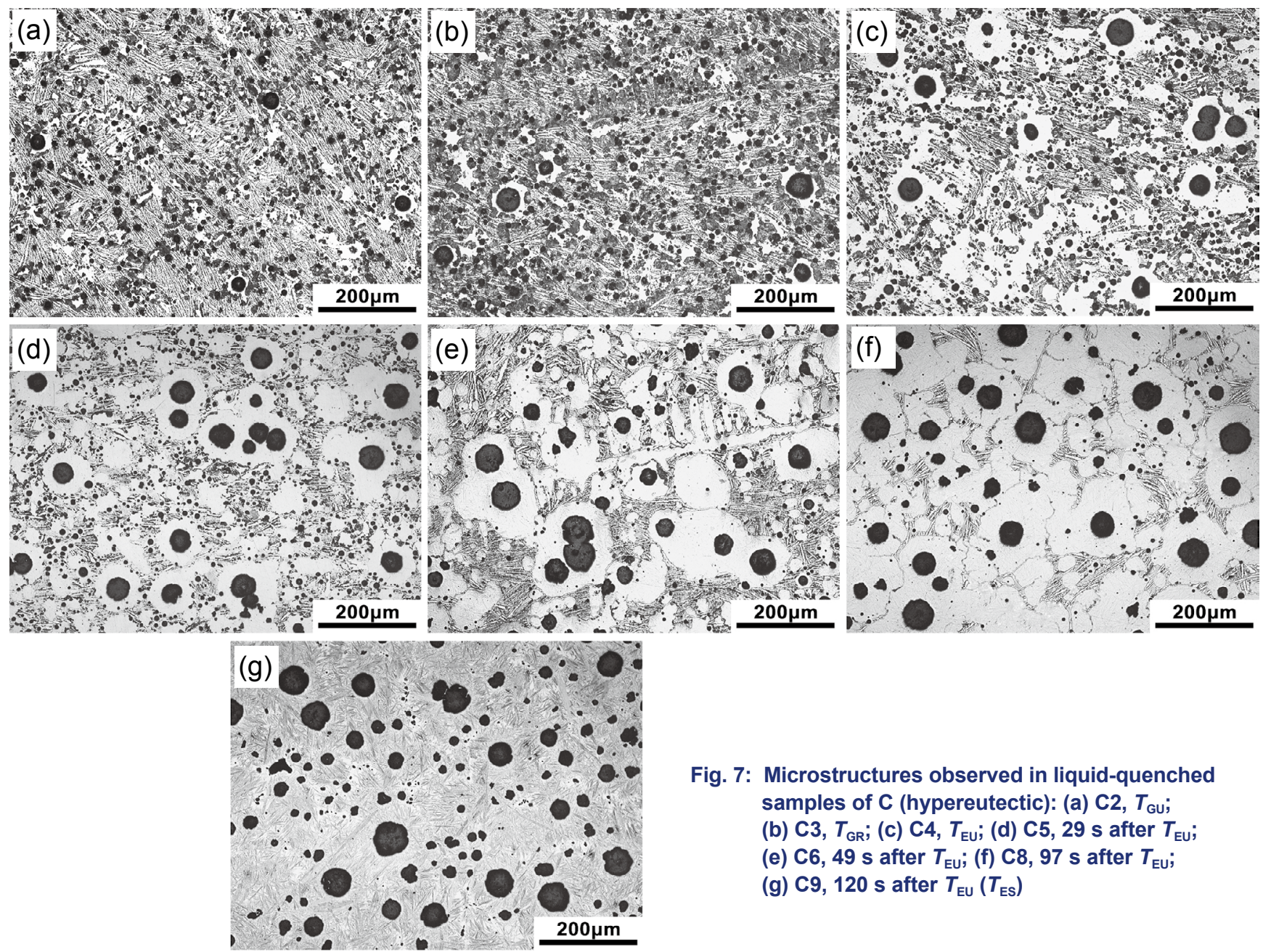

Fig. 7: Microstructures observed in liquid-quenched samples of $C$ (hypereutectic): (a) C2, $T_{\mathrm{Gu}}$; (b) C3, $T_{\mathrm{GR}}$; (c) C4, $T_{\mathrm{EU}}$; (d) C5, $29 \mathrm{~s}$ after $T_{\mathrm{EU}}$; (e) $\mathrm{C} 6,49 \mathrm{~s}$ after $T_{\mathrm{EU}}$; (f) $\mathrm{C} 8,97 \mathrm{~s}$ after $T_{\mathrm{EU}}$; (g) $C 9,120 \mathrm{~s}$ after $T_{\mathrm{EU}}\left(T_{\mathrm{ES}}\right)$

nodules to form big grains, and the rate of eutectic crystallization will slow down. As the eutectic crystallization progressed, the proportion of liquid phase decreased and the liquid was isolated by big grains at the last stage to freeze, as shown in Fig. 7(f). Meanwhile, it became more difficult for carbon atoms in the melt to diffuse into the early-formed graphite. The solidification of the retained melt must be depended on the formation and growth of new nodules. However, there was not enough melt to provide the growth of new nodular graphite, and the diameter of new graphite was smaller. Therefore, the diameters distribution of graphite nodules in hypereutectic ductile iron is not uniform, as shown in Fig. 7(g).

According to analysis of the cooling curves and the microstructure, it can be found that most of the liquid solidifies between $T_{\mathrm{ER}}$ and $T_{\mathrm{ES}}$. The graphite nodules can nucleate directly in the liquid above the $T_{\mathrm{EU}}$ temperature and grow to a certain size. Both hypoeutectic and hypereutectic ductile irons can form the austenite dendrites during solidification. However, their formation mechanisms are different. During the eutectic crystallization, the austenite dendrites and nodules will fuse into larger sized eutectic grains and continue to grow. In the final stage of eutectic crystallization, it is necessary to rely on the formation of new graphite spheres to promote the completion of eutectic crystallization.

\subsection{Evolution of graphite during solidification}

Figure 8 shows the evolution of nodule count, average diameter, graphite fraction and nodularity during the solidification of three kinds of ductile irons. As shown in Fig. 8(a), the nodule count of the three kinds of ductile irons increased gradually with the increase of freezing time. When the solidification is finished, the eutectic cast iron had the highest nodule count, while the hypoeutectic had the least. It can also be seen from Fig. 8(b) and (c) that the average diameter of graphite nodules and graphite fraction increased with the solidification time. However, in the final stage of the solidification, the average diameter of graphite nodules was reduced due to the size of the new nodules being restricted in the retained melt. At the end of solidification, the hypereutectic ductile iron had the largest nodule diameter and the highest graphite fraction; the eutectic ductile iron had the smallest nodule diameter and its graphite fraction was lower than that in hypereutectic ductile iron; the hypoeutectic ductile iron had the smallest graphite fraction, and its size of nodules is between the other two ductile irons.

At the beginning of the solidification, the nodularity of the three ductile irons was relatively high, the nodularity of hypereutectic ductile iron was the highest among them, and the hypoeutectic one was the smallest. The changing trends of the nodularity with the freezing time are different. For 
(a)

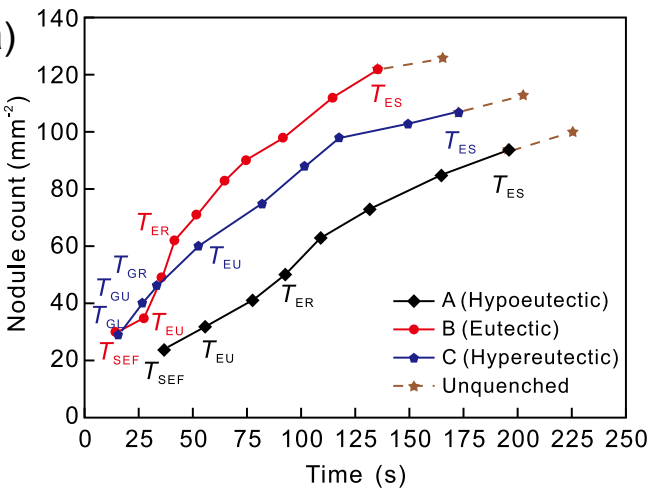

(c)

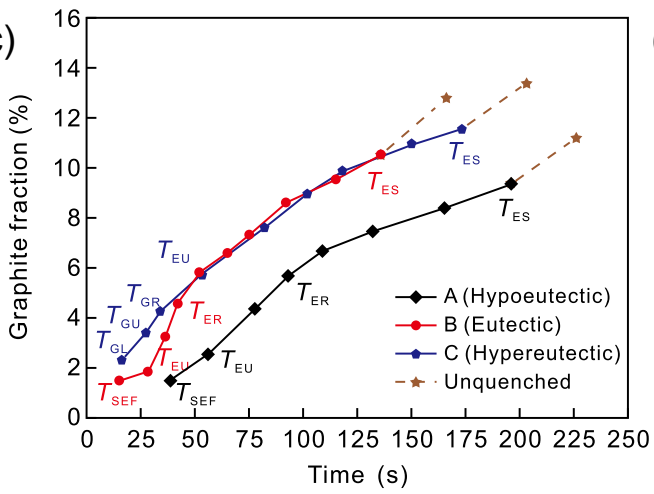

(b)

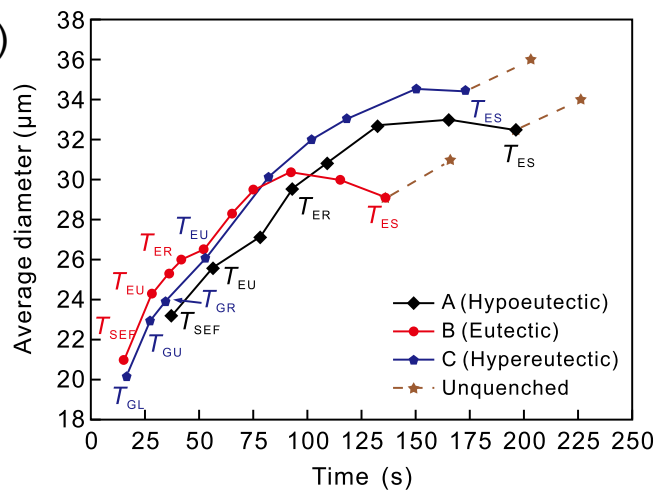

(d)

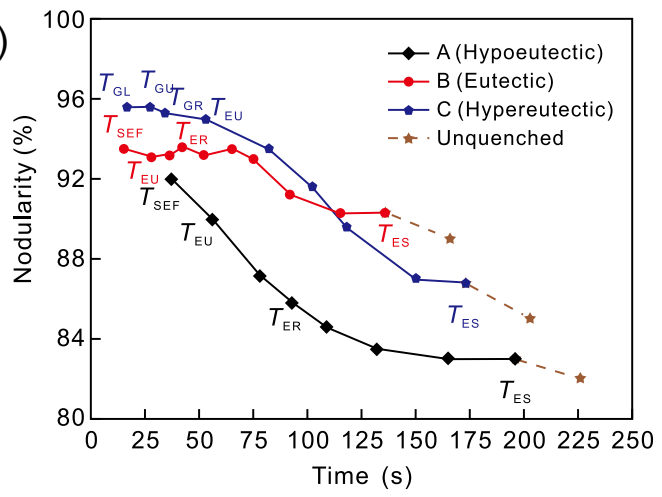

Fig. 8: Evolutions of graphite during the solidification of three kinds of ductile iron: (a) nodule count; (b) average diameter; (c) graphite fraction; (d) nodularity

hypereutectic and hypoeutectic ductile irons, their nodularities all decreased with the progress of solidification, and the nodularity of the hypoeutectic ductile iron decreased more quickly than hypereutectic iron. However, the nodularity of eutectic ductile iron did not change much in the early stage of solidification, and only decreased in the middle stage of the eutectic process. In the final stage of solidification, the nodularity of all the three ductile irons did not change much. Finally, the nodularity of eutectic ductile iron was the highest, the hypoeutectic was the lowest, as shown in Fig. 8(d).

When all the melts were transformed into solid phase, the carbon in the austenite separated out with the decrease of temperature. One part of them, near the graphite nodules, diffused on the surface of the nodules, and the other part was far away from the graphite nodules. It was difficult for them to diffuse into the surface of graphite nodules during rapid cooling, so, they nucleated on the boundaries of austenite grain to form small new nodules ${ }^{[23,30]}$. As a result, the nodule diameter and the volume fraction of graphite increased to some extent. However, the nodularity reduced due to the uneven diffusion of carbon atoms.

\section{Discussion}

The solidification of ductile iron can be classified to two stages, which are the pro-eutectic stage and the eutectic stage. The eutectic crystallization of ductile iron is the most important process from liquid to solid transformation. It determines the microstructure and properties of ductile iron. Therefore, researchers pay great attention to the eutectic crystallization process of ductile iron. In order to easily understand the evolution of nodular graphite during the eutectic process, the $T_{\mathrm{EU}}$ point (bulk eutectic start) was taken as the zero point, and the variation of nodule count, nodularity, volume fraction and precipitation ratio of nodular graphite were studied as functions of eutectic freezing time. The results are shown in Fig. 9.

\subsection{Nucleation rate of eutectic graphite and its effect on nodule count}

According to the data in Fig. 9(a), the nucleation rates of eutectic graphite nodules were calculated within different stages of eutectic crystallization, and a plot of nucleation rate versus eutectic time was obtained, as shown in Fig. 10. It can be seen that the nucleation rate of eutectic graphite nodules of three kinds of ductile irons keep growing after $T_{\mathrm{EU}}$, and then reach the highest value. The maximum nucleation rate of eutectic iron, hypereutectic iron and hypoeutectic iron are about $130 \mathrm{~mm}^{-2} \cdot \mathrm{min}^{-1}, 39 \mathrm{~mm}^{-2} \cdot \mathrm{min}^{-1}$, and $49 \mathrm{~mm}^{-2} \cdot \mathrm{min}^{-1}$, respectively. The nucleation rate is proportional to the fraction of remaining liquid phase. Therefore, with the decrease of residual melt, the nucleation rates decrease after the peak value. At the last stage of eutectic, the temperature of the retained melt becomes lower and the undercooling gradually becomes greater. The driving force of nucleation increased and new nuclei precipitated. Therefore, the nucleation rate does not remarkable decrease, and even increased to some extent.

As for eutectic ductile iron, no primary phases form before the starting of eutectic $\left(T_{\mathrm{EU}}\right)$, all melts solidified in the eutectic crystallization process. When eutectic crystallization stage began, a large number of eutectic graphite nuclei were produced, 
(a)

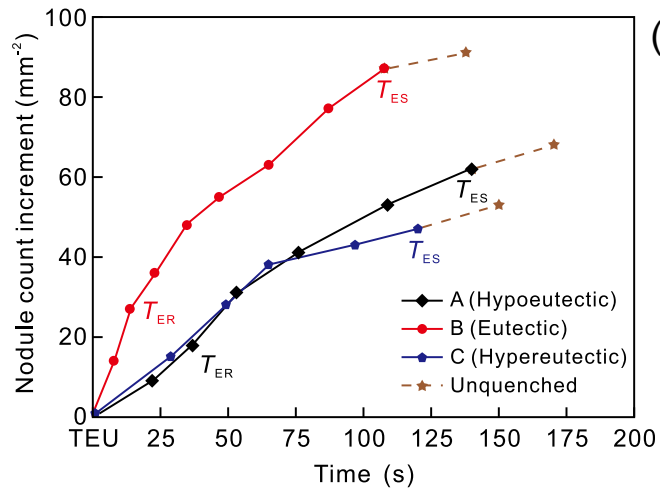

(c)

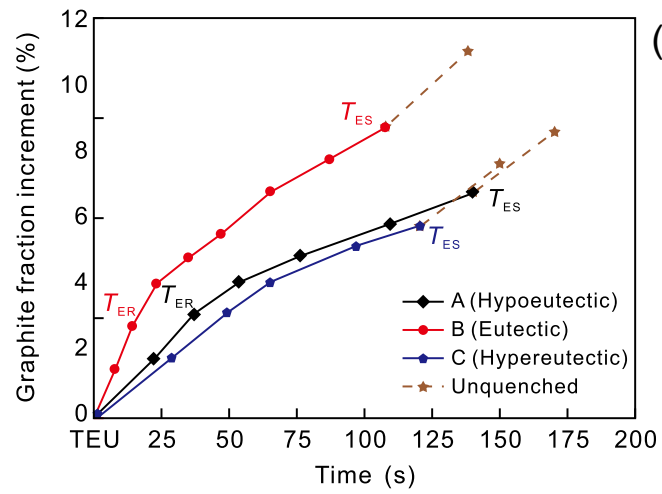

(b)

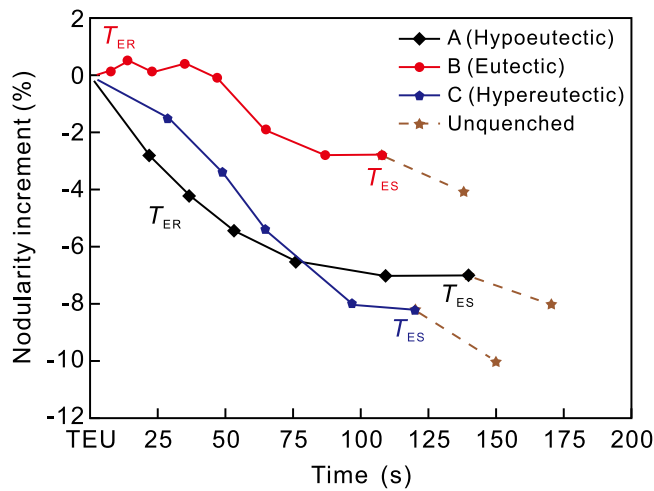

(d)

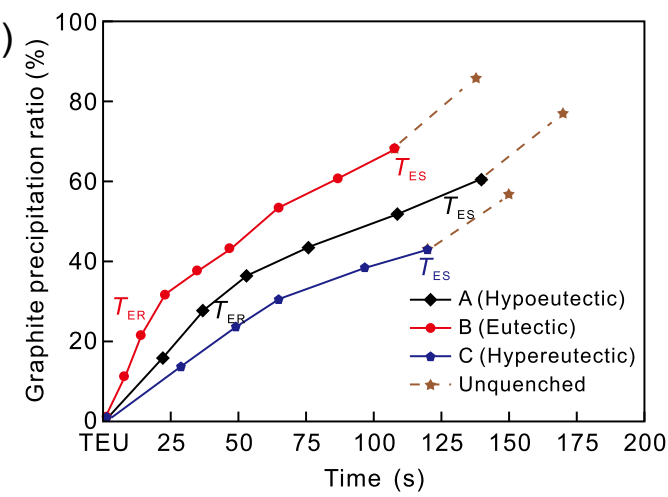

Fig. 9: Evolution of graphite during eutectic crystallization of three kinds of ductile irons: (a) nodule count increment; (b) nodularity increment; (c) graphite fraction increment; (d) graphite precipitation ratio

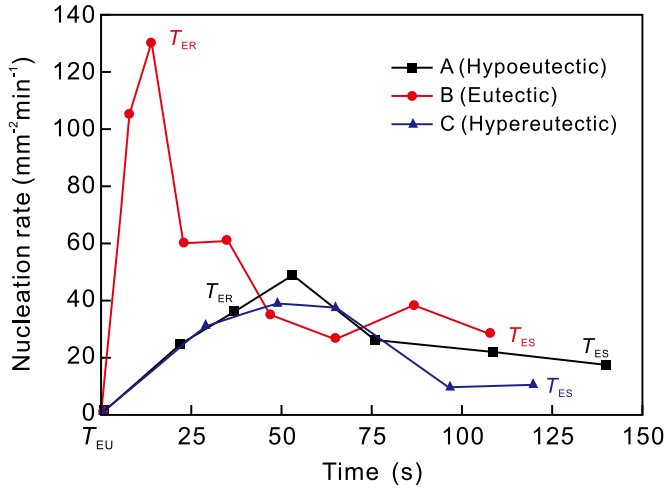

Fig. 10: Nucleation rate of graphite nodules in three ductile irons during the eutectic crystallization stage

and the number of eutectic graphite nodules increased very quickly [Fig. 9(a)]. The hypoeutectic ductile iron has the lowest eutectic nucleation rate due to the formation of primary austenite dendrites, and its eutectic graphite nodules was much less than that of eutectic ductile iron. The hypereutectic melt with a high carbon equivalent contains many more clusters of carbon atoms, which promotes the graphite nucleation. A certain number of primary graphite are produced before eutectic, which can be acted as the nucleation substrates of eutectic crystallization. So, the eutectic crystallization of hypereutectic melt can be performed under the condition of lower undercooling, which weakened the ability of formation of new nucleus. This leads to the lower nucleation rate of graphite during eutectic process. The increment of eutectic graphite nodules was the same as that of hypoeutectic cast iron during earlier stage of eutectic solidification. However, the nucleation rate of hypereutectic cast iron in the last to freeze region is the lowest. Therefore, the increment of eutectic graphite number is the lowest.

\subsection{Effect of austenite dendrites on nodularity}

Previous studies illustrate that austenite dendrites not only exist in hypoeutectic ductile iron but also in eutectic and hypereutectic ductile iron ${ }^{[31,32]}$. In this study, it was found that austenite dendrites have important effects on the nucleation position and growth of graphite nodules, as shown in Fig. 5 and Fig. 7. At the beginning of solidification, the roundness of graphite was very high, but as the eutectic crystallization progresses, the nodularity of the hypoeutectic and hypereutectic ductile iron decreased remarkably, while the nodularity of the eutectic ductile iron still kept a high degree.

For hypoeutectic and hypereutectic ductile irons, a large number of graphite nodules formed between the dendrites, enveloped by austenite shell quickly when eutectic crystallization started. As the graphite nodules grew, they merged with austenite dendrites to form larger grains, and the thickness of their austenite shell became uneven. The uniformity of thickness will further become worsen with the eutectic time, which resulted in un-uniform supply of carbon atoms for nodular graphite in all directions. Therefore, the nodularity of hypoeutectic and hypereutectic ductile irons reduced significantly with the continuing eutectic crystallization.

For eutectic ductile iron, when the eutectic crystallization began, a great number of graphite nodules directly formed in the melt, and then grew in a more uniform austenite shell (Fig. 5). The growth rate in all directions was basically the 
same. Therefore, the nodularity was high in the early stage of eutectic, as shown in Fig. 8(b). But when the eutectic solidification was more than halfway, the thickness of the austenite shell became greater, and the austenite shells merged with each other to form big eutectic grains. In the same way, this would lead to non-uniform diffusion of carbon atoms, and the nodularity of eutectic ductile iron decreased to some extent. However, the reduction was much smaller than that of hypoeutectic and hypereutectic ductile irons.

In the final stage of eutectic, since all the three ductile irons formed many fresh and fine graphite spheres, the average nodularity decreased little. It can be found in Fig. 9(b), at the end of eutectic time, the nodularity of eutectic ductile iron was only reduced $3 \%$ compared with the beginning, while the hypoeutectic and hypereutectic ductile irons were reduced $7 \%$ and $8 \%$, respectively. In summary, the primary austenite dendrites in ductile iron are harmful in obtaining a high nodularity. In addition, it can be seen that the nodularity was further reduced by $1 \%-2 \%$ during the solid phase transformation.

\subsection{Effect of eutectic graphite precipitation on shrinkage}

The formation of shrinkage defects of castings is closely related to the contraction of liquid and solidification, and it also depends on the precipitation volume and the rate of graphite during eutectic solidification. Figure 11 shows the growing rate of graphite fraction in ductile iron during different stages of eutectic solidification. The density of austenite is greater than that of the melt. When the austenite precipitated from the melt, the solidifying shrinkage occurred. Because the density of graphite is less than $1 / 3$ that of the melt, the formation of graphite will bring expansion, which could compensate for a part of liquid and solidification shrinkage ${ }^{[33,34]}$. Traditionally, it is believed that the higher carbon content of ductile iron will produce a greater expansion, which is more helpful in eliminating the shrinkage defects of castings. However, what is confusing is that when the carbon equivalent of ductile iron is too high, the shrinkage defect increases ${ }^{[33]}$. Actually, the shrinkage is not only related to the total volume of graphite precipitation, but also to the precipitation rate at different stages.

As shown in Fig. 8c, Fig. 9c and Fig. 11, the precipitation

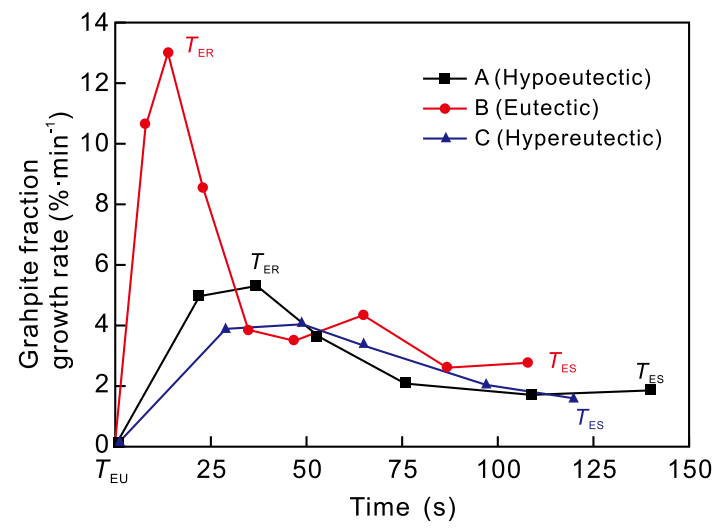

Fig. 11: Graphite fraction growth rate of three ductile iron during the eutectic crystallization behavior of graphite in the three kinds of ductile irons was significantly different. In the period of eutectic, the growth rate of graphite fraction increases rapidly in the beginning, afterwards decreases in the middle. Finally, the rate does not change remarkably. In this study, the total graphite fraction of hypoeutectic, eutectic, and hypereutectic ductile iron was $11.2 \%, 12.8 \%$ and $13.4 \%$, respectively. And a part of graphite had already precipitated before eutectic, of which hypereutectic ductile iron is about $5.8 \%$ ( $43 \%$ of the total graphite), the hypoeutectic about $2.6 \%$ (23\% of the total graphite), and the eutectic about $1.8 \%$, only accounting for $14 \%$ of the total graphite.

When the primary graphite precipitates before eutectic, the casting has a high liquid phase fraction, and its liquid shrinkage can be compensated for from the gating and riser system. In contrast, the expansion caused by graphite precipitation at this time will cause the melt to flow back into the riser. Moreover, if the riser neck is frozen and the rigidity of mold is low, it will also cause the mold cavity to expand, which affects the dimensional accuracy of castings ${ }^{[35,36]}$. On the other hand, the formation of primary graphite will result in the decrease of eutectic graphite precipitation, and reduce the ability of eutectic self-feeding. It is concluded that the more the pro-eutectic graphite precipitates, the more serious the shrinkage defects in ductile iron castings.

Researchers have proven that the graphite growth during the eutectic crystallization, especially at the last period, contributes a lot to the elimination of shrinkage porosity ${ }^{[25,37]}$. It can be seen from Fig. 9(c) and d that with the progress of eutectic, the graphite fraction of the three kinds of ductile irons gradually increased. During the eutectic crystallization stage, the eutectic ductile iron had the largest amount of graphite precipitation, up to $8.7 \%$, accounting for $68 \%$ of the total graphite, the hypereutectic ductile iron had the least graphite precipitation at $6 \%$, accounting for $43 \%$ of the total graphite, and the hypoeutectic ductile iron was in the middle at $7 \%$, accounting for $61 \%$ of the total. Therefore, the eutectic composition ductile iron has the least shrinkage tendency.

In addition, after the end of solidification, as the temperature decreases, the carbon in the austenite precipitates to allow the graphite nodules to continue to grow. The graphitization expansion also happens at solid state. It is helpful to eliminate the intergranular micro porosity. Because the nodule count of eutectic ductile iron is the highest and their distribution is the most uniform, the diffusion distance of carbon atoms is shorter from austenite to surface of nodular graphite. The secondary graphitization of eutectic iron is easier and more than that of hypo and hyper eutectic irons. As a result, the graphite fraction of the secondary precipitation of eutectic iron is higher, about $2.3 \%$, more than that of hypoeutectic $(1.8 \%)$ and hypereutectic iron $(1.7 \%)$.

Figure 12 shows the shrinkage of hypoeutectic, eutectic and hypereutectic unquenched samples without riser and thermocouple. For hypoeutectic and hypereutectic samples, there are bigger surface buckling defects and many porosities 

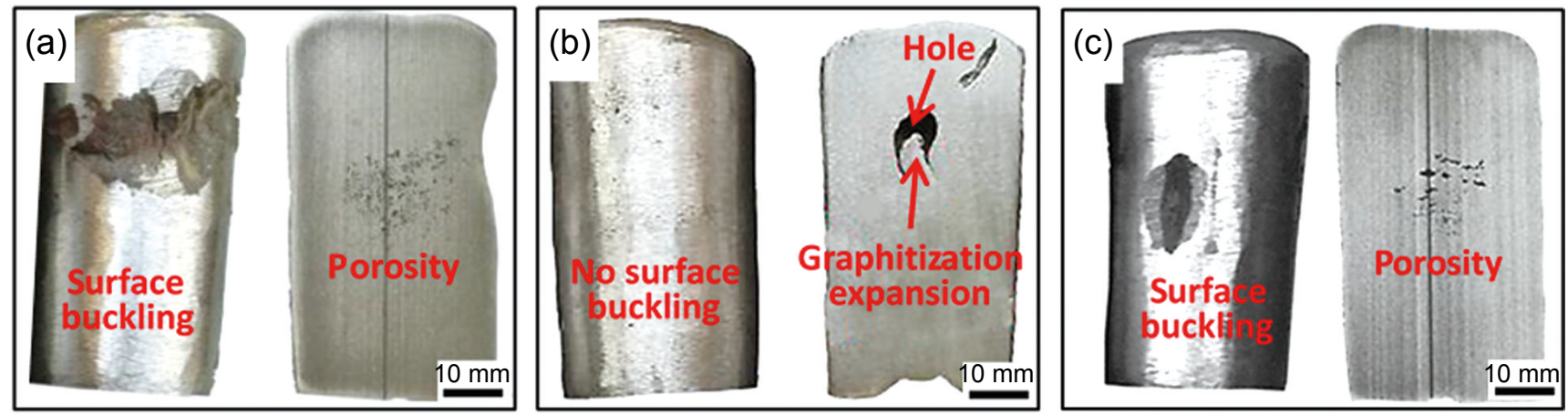

Fig. 12: Shrinkage of unquenched samples: (a) A (Hypoeutectic); (b) B (Eutectic); (c) C (Hypereutectic)

inside. Because ductile iron has the characteristic of mushy solidification, a solid skin layer of sample cannot form sooner. Due to the precipitation of much pre-eutectic austenite, the liquid contracts greatly in the earlier stage of solidification, which leads to a decrease of liquid volume inside the sample. An inner vacuum may be formed. Under the action of atmospheric pressure, the surface buckling will happen. Also, owing to less volume fraction of eutectic graphite, the graphitizing expansion is small and the ability of self-feeding is reduced. This results in the formation of much porosity inside sample.

There is no surface buckling on the eutectic ductile iron sample except a small shrinkage hole. In addition, the shrinkage hole has a bulge due to the great graphitizing expansion during the eutectic solidification process. During the last stage of eutectic, the retained melt was squeezed to the shrinkage cavity to form the bulge and reduce the shrinkage. If a suitable liquid compensation is provided for the sample, there will be no shrinkage defects inside the eutectic sample. However, for hypo and hyper eutectic samples, even if some of the liquid compensation is designed, it cannot ensure the production of a compact sample.

\section{Conclusions}

(1) The first nucleus of nodular graphite in ductile iron directly forms in the liquid above the $T_{\mathrm{EU}}$ temperature and grows to a certain size. The count, diameter and volume fraction of nodular graphite increase with the increase of solidification time, while the nodularity decreases gradually.

(2) The nucleation rate, nodularity and expansion rate of graphite are the greatest at the beginning stage of eutectic crystallization, then decrease and change unremarkably at the end. The eutectic graphitizing ratio in eutectic ductile iron is the largest. Therefore, the self-feeding ability of eutectic ductile iron is the best among three kinds of ductile iron.

(3) The precipitation and growth of austenite dendrites in hypoeutectic and hypereutectic ductile irons are harmful to obtain a high nodularity, and also significantly increase the shrinkage tendency of the castings.

(4) After the solidification, the nodule count and nodularity of eutectic ductile iron are the highest, and that of hypoeutectic ductile iron are the lowest, and that of the hypereutectic are in the middle. The diameter and volume fraction of nodular graphite in hypereutectic ductile iron are the largest. The nodules diameter of eutectic cast iron and the graphite fraction of hypoeutectic cast iron are the smallest. The graphite diameter of hypoeutectic and the graphite fraction of eutectic are in between

(5) In the process of solid transformation, the nodularity of graphite in ductile iron decreases, and the count, diameter, and graphite fraction of nodular graphite increase to some extent.

\section{Acknowledgments}

This work was financially supported by the National Natural Science Foundation of China (NSFC) under Grant Nos. 51601054 and 51775006, the Natural Science Foundation of Hebei Province of China under Grant Nos. E2017202095 and E2016202100, the Scientific and Technological Transformative Project of Tianjin Supporting Beijing-Tianjin-Hebei under Grant No. 18YFCZZC00030, and the Central Government Guides Local Science and Technology Development Fund Projects under Grant No. 206Z1005G.

\section{References}

[1] Zhou J Y. Colour metallography of cast iron: spheroidal graphite cast iron (I). China Foundry, 2010, 7(1): 76-88.

[2] Labrecque C, Gagne M. Review ductile iron: Fifty years of continuous development. Canadian Metallurgical Quarterly, 1998, 37(5): 343-378.

[3] Liu J H, Xiong P, Fu B G, et al. Effects of austempering temperature on microstructure and surface residual stress of carbidic austempered ductile iron (CADI) grinding balls. China Foundry, 2018, 15(3): 173-181.

[4] Fu B G, Li Z Q, Zhao X B, et al. Formation mechanism of spheroidal carbide in ultra-low carbon ductile cast iron. China Foundry, 2016, 13(5): 346-351.

[5] Zimba J, Simbi D J, Navara E. Austempered ductile iron: an alternative material for earth moving components. Cement and Concrete composites, 2003, 25(6): 643-649.

[6] Qing, J J, Richards V L, Van Aken D C. Examination of spheroidal graphite growth and austenite solidification in ductile iron. Metallurgical and Materials Transactions A, 2016, 47: 6197-6213. 
[7] Čanžar P, Tonković Z, Kodvanj J. Microstructure influence on fatigue behaviour of nodular cast iron. Materials Science and Engineering: A, 2012, 556: 88-99.

[8] Liu J C, Elliott R. The influence of cast structure on the austempering of ductile iron. Part 3 . The role of nodule count on the kinetics, microstructure and mechanical properties of austempered $\mathrm{Mn}$ alloyed ductile iron. International Journal of Cast Metals Research, 1999, 12(3): 189-195.

[9] Lekakh S N, Hrebec B. Solidification Kinetics of Graphite Nodules in Cast Iron and Shrinkage Porosity. International Journal of Metalcasting, 2016, 10(4): 389-400.

[10] Górny M, Kawalec M, Sikora G, et al. Primary structure and graphite nodules in thin-walled high-nickel ductile iron castings. Metals, 2018, 8(8): 649 .

[11] Stefanescu D M, Alonso G, Larrañaga P, et al. A comparative study of graphite growth in cast iron and in analogous systems. International Journal of Metalcasting, 2018, 12(4): 722-752.

[12] Lacaze J. Trace elements and graphite shape degeneracy in nodular graphite cast irons. International Journal of Metalcasting, 2017, 11(1): 44-51.

[13] Natxiondo A, Suárez R, Sertucha J, et al. Graphite and solid fraction evolutions during solidification of nodular cast irons. Metals, 2015, 5(1): 239-255.

[14] Stefanescu D M, Suarez R, Kim S B. 90 years of thermal analysis as a control tool in the melting of cast iron. China Foundry, 2020, 17(2): 69-84.

[15] Stefanescu D M. Solidification and modeling of cast iron-A short history of the defining moments. Materials Science and Engineering: A, 2005, 413-414: 322-333.

[16] Stefanescu D M. Thermal analysis-theory and applications in metalcasting. International journal of metalcasting, 2015, 9(1): $7-22$.

[17] Stefanescu D M, Alonso G, Larrañaga $P$, et al. On the crystallization of graphite from liquid iron-carbon-silicon melts. Acta Materialia, 2016, 107: 102-126.

[18] Stefanescu D M, Alonso G, Larrañaga P, et al. On the stable eutectic solidification of iron-carbon-silicon alloys. Acta Materialia, 2016, 103: 103-114.

[19] Alonso G, Larrañaga $P$, Stefanescu D M, et al. Kinetics of nucleation and growth of graphite at different stages of solidification for spheroidal graphite iron. International Journal of Metalcasting, 2017, 11(1): 14-26.

[20] Fredriksson $\mathrm{H}$, Stjerndahl J, Tinoco J. On the solidification of nodular cast iron and its relation to the expansion and contraction. Materials Science and Engineering: A, 2005, 413414: 363-372.

[21] Liu J H, Yi L T, Li G L, et al. Influence of fading on characteristics of thermal analysis curve of compacted graphite iron. China Foundry, 2011, 8(3): 295-299.
[22] GB/T 9441-2009. Metalllographic test for spheroidal graphite cast iron. Standards press of China, 2009.

[23] Chang S, Shangguan D, Stefanescu D M. Modeling of the liquid/solid and the eutectoid phase transformations in spheroidal graphite cast iron. Metallurgical and Materials Transactions A, 1992, 23(4): 1333-1346.

[24] Wang C Q. The uneven concentration inoculation theory. In: Theory and practice of inoculation of cast iron. Tianjin: Tianjin university press, 1991: 122-138. (In Chinese)

[25] Zhou J Y. Colour metallography of cast iron: spheroidal graphite cast iron (III). China Foundry, 2010, 7(3): 292-307.

[26] Lesoult G, Castro M, Lacaze J. Solidification of spheroidal graphite cast irons-I. Physical modelling. Acta materialia, 1998, 46(3): 983-995.

[27] Zhou J Y. Colour metallography of cast iron: introduction (I). China Foundry, 2009, 6(1): 57-69.

[28] Pedersen K M, Tiedje N. Nucleation and solidification of thin walled ductile iron-experiments and numerical simulation. Materials Science and Engineering: A, 2005, 413-414: 358-362.

[29] Rivera G, Boeri R, Sikora J. Influence of the inoculation process, the chemical composition and the cooling rate, on the solidification macro and microstructure of ductile iron. International Journal of Cast Metals Research, 2003, 16(1-3): 23-28.

[30] Carazo F D, Dardati P M, Celentano D J. Stable eutectoid transformation in nodular cast iron: Modeling and validation. Metallurgical and Materials Transactions A, 2017, 48A: 63-75.

[31] Xu J F, Zhai Q Y, Yuan S, et al. Morphology characteristic and growing manner of austenite in hypereutectic nodular iron during the solidification process. Acta Metallurgica Sinica, 2003, 39(2): 136-139. (In Chinese)

[32] Rivera G L, Boeri R E, Sikora J A. Revealing the solidification structure of nodular iron. Cast Metals, 1995, 8(1): 1-5.

[33] Anjos V, Silva Ribeiro C A S. Maximization and control of nodular iron melt's self-feeding characteristics to minimize shrinkage. Materials Science Forum, 2018, 925: 147-154.

[34] Jimbo I, Cramb A W. The density of liquid iron-carbon alloys. Metallurgical and Materials Transactions B, 1993, 24B: 5-10.

[35] Kanno T. Effect of pouring temperature, composition, mould strength and metal flow resistance on shrinkage cavities in spheroidal graphite cast iron. International Journal of Cast Metals Research, 2008, 21(1-4): 2-6.

[36] Chisamera M, Riposan I, Stan S, et al. Shrinkage evaluation in ductile iron as influenced by mould media and inoculant type. International Journal of Cast Metals Research, 2011, 24(1): 28-36.

[37] Erturk S, Kumruoglu L, Ozel A. Determination of feederless casting limits by thermal analysis in cast iron. Acta Physica Polonica A, 2017, 131(3): 370-373. 